\title{
Can meaningful effective connectivities be obtained between auditory cortical regions?
}

\footnotetext{
* Miguel S. Gonçalves ${ }^{\text {CA }},{ }^{*}$ Deborah A. Hall, ${ }^{\dagger \ddagger}$ Ingrid S. Johnsrude, ${ }^{*}$ Mark P. Haggard

*MRC Institute of Hearing Research

${ }^{\dagger}$ MRC Cognition and Brain Sciences Unit

University Park

15 Chaucer Road

Nottingham

Cambridge

U.K.

U.K.

NG7 2RD

CB2 2EF
}

${ }^{\ddagger}$ The Functional Imaging Laboratory

Wellcome Department of Cognitive Neurology

St Johns House

12 Queen Square

London

U.K.

WC1N 3BG

CA corresponding author

Tel: (+44) 1159223431

Fax: (+44) 1159518503

email:miguel@ihr.mrc.ac.uk

Date of Submission: 15/03/2001

Running Title: SEM of Auditory Networks 


\begin{abstract}
Structural equation modelling (SEM) of neuroimaging data can be evaluated both for the goodness of fit of the model and for the strength of path coefficients (as an index of effective connectivity). SEM of auditory fMRI data is made difficult by the necessary sparse temporal sampling of the time series (to avoid contamination of auditory activation by the response to scanner noise), and by the paucity of well-defined anatomical information to constrain the functional model. We used SEM (i.e. a model incorporating latent variables) to investigate how well fMRI data in four adjacent cortical fields can be described as an auditory network. Seven out of 14 models ( 2 hemispheres x (6 subjects and 1 group)) produced a plausible description of the measured data. Since the auditory model to be tested is not fully validated by anatomical data, our approach requires that goodness of fit must be confirmed to assure generalisability of connectivity patterns. For good-fitting models, connectivity patterns varied significantly across subjects and were not replicable across stimulus conditions. SEM of central auditory function therefore appears to be highly sensitive to the voxel-selection procedure and/or the sampling of the time series.
\end{abstract}

Key words: Structural Equation Modelling; Auditory fMRI, Model goodness of fit; Intersubject variability; Sparse temporal sampling. 


\section{Introduction}

Structural equation modelling (SEM) can quantify interactions among multiple brain areas (McIntosh and Gonzalez-Lima, 1993) and permit direct inferences to be drawn about the functioning of whole networks that can only be hypothesised from straightforward mapping results. SEM of imaging data usually reports the results from either a group analysis (McIntosh and Gonzalez-Lima, 1991; McIntosh and Gonzalez-Lima, 1993; McIntosh and Gonzalez-Lima, 1994a; Horwitz et al., 1995; McIntosh and Gonzalez-Lima, 1995; Jennings et al., 1998; Bullmore et al., 2000), or an individual subject analysis (Büchel and Friston, 1997) and tends to seek confirmation of hypothesised patterns of task-specific connectivity rather than to test model generalisability. There is more than one epistemological approach to SEM and a greater body of neuroimaging data is required for a fair critical assessment between them. Most of the SEM applications in neuroimaging research have so far tested for condition-specific differences regardless of the model fit, since the true connections between the different neuroanatomical areas are assumed to be known (e.g., refMacIntosh 94). However, in cases where the neuroanatomical model is not well defined, one could equally adopt the alternative strategy that has been widely applied in behavioural science. This approach seeks to confirm that a model accounts for as much of the variance as possible in order to confirm the proposed anatomical network, prior to evaluating changes in path connections with experimental condition. This strategy is based on that described by Bentler (1992) and MacCallum (1995) for testing behavioural data and is the preferred way to test models that have not been independently validated. In the present study, we take the position that good model fits are important for the interpretation of connectivity because current anatomical knowledge does not permit the prior full specification of the auditory cortical network. Neuroimaging data provides a relatively recent application for the well-established statistical technique of SEM, yet SEM in functional magnetic resonance imaging (fMRI) has 
been applied to study human learning (Büchel et al., 1999), semantic decision (Bullmore et al., 2000) and the modulation of visual processing by attention (Büchel and Friston, 1997). All these studies use widely distributed networks over the brain, where there is prior functional anatomical knowledge to suggest the role each linked area might play. Within less widely distributed networks, such as the visual system, there may be a similar opportunity, as functional distinctions are well mapped (Hadjikhani et al., 1998). In principle, therefore, SEM should be useful in revealing inter-relationships between auditory areas. However, in both the general and the particular context of SEM, auditory fMRI faces two major challenges not encountered in other domains.

Firstly, the background scanner noise induces additional auditory activation that is unrelated to any stimulus or task manipulation of interest. This acoustical interference can be eliminated by increasing the interscan interval to introduce a quiet period during which stimuli are presented. However, such sparse temporal sampling generally permits the acquisition of rather few data points per epoch, usually in a fixed position relative to stimulus onset (Hall et al., 2000b). Sampling relatively few fixed points on the response curve limits the variance of the response across which inter-correlations can be explored. This decreases the temporal resolution of the fMRI time series and reduces the residual degrees-of-freedom required to support a model for effective connectivity. In turn, the likelihood of rejecting the model is increased because the approximations to the chi-square distribution may be unreliable (Bullmore et al., 2000).

A second difficulty arises from the paucity of knowledge about the functional anatomy of human auditory cortex, especially for non-primary fields. The critical issue is that the number, location, and inter-connections of the multiple non-primary fields have been little studied in humans (Galaburda and Sanides, 1980; Rivier and Clarke, 1997). Moreover, the borders of these regions vary across individuals. This lack of specific localisation hinders 
both model definition and the appropriate selection of voxels to represent each functional field for fitting time-course data using SEM. Any suggested auditory cortical network is only partly validated by anatomical information. As a consequence, we adopt a confirmatory strategy (as defined by MacCallum, 1995) in which we first confirm that our model accounts for as much of the variance as possible, before we begin to make informative inferences about the model (i.e., the nature of the condition-specific connectivities).

In the present dataset, preferential responses to tone stimuli have been localised with reference to estimates of anatomically defined auditory areas (Hall et al., 2000a). Frequencymodulated (FM) tones activate dorsolateral regions of the superior temporal gyrus more than do static tones (Hall et al., 2000a). Our hypothesis is that modulation should therefore also influence path connections among those areas that show response selectivity for modulation. Given the uncertainty about functional borders, we evaluate whether the voxel-selection procedure is critical for both the goodness-of-fit of the SEM and the interpretation of path coefficients, as one form of generalisation test. We report the results for both group and individual voxel-selection procedures to determine the degree of consistency between subjects.

\section{Materials and Methods}

Subjects: We employed six subjects aged 28-49 (subjects 4 and 5 were female). Subject 4 was left-handed. All were neurologically normal, without hearing impairment, and gave informed written consent.

Stimuli and Task Design: Four acoustical stimuli were defined by crossing two types of carrier tones with two types of modulation. The carrier was either a single $500 \mathrm{~Hz}$ tone or a harmonic-complex tone with components at 186, 372, 558, 744, 930, and $1116 \mathrm{~Hz}$. Stimuli were 900-ms long, were either static or cosinusoidally frequency modulated at $5 \mathrm{~Hz}$. They 
were diotically presented over high-fidelity electrostatic headphones (Palmer et al., 1998). Tones were equated for loudness (Moore et al., 1997) by presenting at $94 \mathrm{~dB}$ SPL (single tones) and $84 \mathrm{~dB}$ SPL (harmonic-complex tones). Each trial lasted $16 \mathrm{~s}$ and comprised a series of 15 tone bursts separated by 100 ms periods of silence. To maintain attention, occasional tones were $1900 \mathrm{~ms}$ long and subjects were instructed to press a button whenever this occurred. Tone conditions were presented in a counterbalanced order with a silent resting baseline occurring at every fifth trial. Subjects were scanned in two 32-minute runs, each run containing 24 trials of each stimulus condition. Thus, in total, 96 multi-image volumes were acquired per condition.

fMRI scanning and data analysis: The experiment was performed on a $2 \mathrm{~T}$ Magnetom VISION (Siemens, Erlangen) whole-body MRI system equipped with a head volume coil. Image volumes were acquired in an oblique axial plane, aligned parallel to the Sylvian fissure $(\mathrm{TR}=8 \mathrm{~s} ; \mathrm{TE}=40 \mathrm{~ms} ; 64 \times 64$ pixels; 3 × 3 × 2.5 mm voxel size; $0.5 \mathrm{~mm}$ inter-slice gap; 30 images per volume; volume acquisition time $=2 \mathrm{sec}$ ). A 5-cm wide coronal saturation band was applied across the eyeballs and frontal poles to null the high-variance MR signal from the eyes. Image volumes were obtained at 8 and 16 s post-stimulus onset.

Data were analysed using SPM99 software (http://www.fil.ion.ucl.ac.uk/spm/). The first three volumes in each run were discarded prior to statistical analysis. For each subject, the remaining volumes for the two runs were realigned to the first volume in the sequence. A structural MRI scan (1 x 1 x $1.5 \mathrm{~mm}$ voxel size) was co-registered to the mean functional image and then images were transformed into standard brain space. These normalised images were spatially smoothed using a Gaussian kernel of $8 \mathrm{~mm}$ width (FWHM) for the group analysis and of $4 \mathrm{~mm}$ for the individual analyses. Low frequency respiratory and cardiac aliasing were removed by high pass filter at 0.38 cycles per minute. 
Analyses were performed by modelling the five stimulus conditions using the General Linear Model. The group analysis was computed as a conjunction of t-contrasts across all subjects ( $\mathrm{p}<0.01$, uncorrected). Each t-contrast was specified by a linear contrast of the parameter estimates for each tone condition relative to the baseline. For each individual, the response to the four tone conditions relative to the silent baseline was tested using an Fcontrast of the parameter estimates. Again, auditory activation was defined by those voxels that exceeded a probability threshold of $\mathrm{p}<0.01$, uncorrected.

Model specification: The tones elicited bilateral auditory activation in all subjects (Hall et al., 2000a). These basic sounds were treated equally by the two hemispheres and, in primary and surrounding secondary auditory areas, generated symmetrical activation that was not affected by handedness or gender. Therefore, we used the same model for both left and right hemispheres and for all subjects. The anatomical model specified four key centres of activation in each hemisphere; (i) the primary auditory region on Heschl's gyrus (HG), (ii) an area that was posterior and lateral to HG, located on the planum temporale (PT), (iii) a portion of the anterolateral superior temporal gyrus (alSTG), and (iv) the dorsal bank of the superior temporal sulcus (STS), inferior to these above auditory areas (see Fig. 1). Selected voxels for model testing were located within each of these four key areas.

Anatomical tracing studies in non-human primates indicate that lateral areas, possibly including parts of PT and alSTG, receive connections from the primary area (Hackett et al., 1996). Thus, the model incorporates connections HG $\rightarrow$ PT and HG $\rightarrow$ alSTG. STS is a multi-modal area that receives dense projections from adjacent lateral belt regions (Hackett et al., 1996) and so we include the connections PT $\rightarrow$ STS and HG $\rightarrow$ STS. The inclusion of a further connection, alSTG $\rightarrow$ PT, significantly improved model fits. Anatomical studies suggest that callosal connections link one auditory field to its contralateral counterpart (e.g., Fitzpatrick and Imig, 1980). However, for diotic sound presentation, where the same auditory 
signal reaches both ears and hence both hemispheres, there will be little informative interaction between hemispheres. This view of predominantly independent hemispheric processing was supported by the results of a unitary model, connecting both hemispheres, which showed that these callosal paths were not significantly different from zero for any individual subject or indeed the group analysis. Therefore, we report the results for each hemisphere modelled separately.

**Figure $1 * *$

Structural Equation Modelling: We used a latent variable model which implies that what is measured is a function of true brain activity, plus some measurement error (McIntosh and Gonzalez-Lima, 1994b). Following Horwitz et al. (1995), activation in each area was summarised by the time course of the most significantly activated voxel and this defined the observed variables in the model. These peak voxels were selected in two ways: using a group-based procedure (involving a conjunction of t-contrasts across subjects), and a procedure based on single subject analyses. A latent variable was derived for each area using the peak voxel, by fixing the error term for the observed and latent variables each at $50 \%$ of the total variance (Hayduk, 1987; McIntosh and Gonzalez-Lima, 1994b). The value of the error term determines how much of the variance in the data is to be explained by the model and can range from 35-80\% (McIntosh and Gonzalez-Lima, 1994b; Bullmore et al., 2000). Four different error values (30\%, 40\% 50\% and 80\%) were tested, but no meaningful changes in the goodness-of-fit of the models or in the path coefficients were found relative to the $50 \%$. The models for the $50 \%$ error term are reported here. Fixing error values at zero can reduce success in achieving a unique solution, whilst leaving the parameters free to vary can lead to indeterminacy in the final solution (McIntosh and Gonzalez-Lima, 1991; McIntosh and Gonzalez-Lima, 1994b). 
The SEM models were applied to the inter-regional correlation matrices within each stimulus condition using the stacked model approach (McIntosh et al., 1994). Stacking each stimulus condition provides a single model estimate. Stacked modelling permits the evaluation of different models, and also improves the goodness of fit by increasing the degrees of freedom in the model (Hayduk, 1987). For the group-based approach, the data for the six subjects were considered as one time course, so giving a generalised model fit.

Model fits and path coefficients for each of the inter-connections, based on the correlations among brain areas, were calculated using maximum likelihood estimation (MLE) implemented in AMOS (Version 4, James L. Arbuckle). Goodness-of-fit was assessed on the fully unconstrained models using $\chi^{2}$, Akaike Information Criterion (AIC) and Root Mean Square Error of Approximation (RMSEA) (Raykov and Widaman, 1995; Haugton et al., 1997). These three measures express different criteria for model goodness of fit (Jaccard and Wan, 1996). The $\chi^{2}$ index is based on the difference between predicted and observed covariances. RMSEA also reflects differences in covariance, whilst penalising for lack of parsimony; AIC is based on parsimony within a wider view of information theory.

Given that the meaning of significant path coefficients in poorly-fitting models is unclear (MacCallum, 1995), we required the fully-unconstrained model to first be a good fit according to the above three measures. To assess the significance of condition-specific changes in path coefficients, we tested the model, with the constraint of interest, against the fully unconstrained model, by taking the difference of their $\chi^{2}$ criteria (denoted $\chi_{\text {diff). This }}^{2}$ statistic allowed us to determine which of the path coefficients were either equal or different between stimulus conditions (McIntosh and Gonzalez-Lima, 1994b). A bootstrapped standardised solution with replacement was used to evaluate whether the path coefficients differed significantly from zero. The standardised solutions facilitate the between-stimuli and between-subjects comparison, since the magnitudes range from zero to one. The bootstrapped 
sample size was the same as the original sample. Bootstrapping defines the $95 \%$ confidence intervals for the path coefficients, and offers a way to assess the stability of Maximum Likelihood Estimates for the original sample. Pre-processing with a partial correlation procedure for removing within-subjects variation due to multiple observations (McIntosh and Gonzalez-Lima, 1991; McIntosh and Gonzalez-Lima, 1994a) did not give significantly different results from the above method.

\section{Results}

Location of peak voxels: Individual analyses revealed inter-subject variation in the locations of the peak of activation. In HG in both hemispheres, the mean variation in the peak locations among subjects was $11 \mathrm{~mm}$ (range $=4$ to $21 \mathrm{~mm}$ ). Shifts in the peak were slightly greater for the larger areas, alSTG and PT, where the peak locations were $15 \mathrm{~mm}$ and $17 \mathrm{~mm}$ apart respectively (ranges for alSTG $=5$ to $36 \mathrm{~mm}$, and for PT $=4$ to $34 \mathrm{~mm}$ ). Peak locations for STS activation displayed the greatest inter-subject variability (e.g. $46 \mathrm{~mm}$ between subjects 4 and 5). Given that the group analysis summarises the data from the 6 subjects, it was unsurprising that peak coordinates identified by the group-based analysis differed slightly from those identified by each individual analysis, but fell within their range.

Model fits: For the group-based voxel selection approach, a good model fit $\left(\chi^{2}=2.05, \mathrm{p}=\right.$ 0.84) was obtained for the right hemisphere data for the fully-unconstrained model. For the left hemisphere, the data differed from the unconstrained model $\left(\chi^{2}=77.11\right.$, $\left.\mathrm{p}<0.01\right)$, thus yielding a poor fit. For the individual-based voxel selection procedure, the data for five subjects fit the model well ( $p>0.05$ ) in at least one hemisphere (subjects 3, 4 and 6 in the right hemisphere and subjects 2, 4 and 5 in the left hemisphere). The RMSEA and the AIC agreed with the $\chi^{2}$ indices. 
Condition-specific effects: Table 1 shows the condition-specific, bootstrapped, standardised path coefficients for well-fitting models. The significance of condition-specific connectivities was assessed relative to all other conditions.

The group-based modelling approach indicated that for the right hemisphere, path HG $\rightarrow$ PT was higher for the static harmonic-complex condition $\left(\chi^{2}\right.$ diff,1 $\left.=5.09, \mathrm{p}=0.02\right)$ and that path PT $\rightarrow$ STS was lower for the static harmonic-complex and single tone conditions $\left(\chi^{2}\right.$ diff, 1 $=15.36, \mathrm{p}<0.01$ ) than for the other conditions.

$* *$ Table $1 * *$

The individual-based approach indicated some inter-subject differences in conditionspecific connectivities. For subject 2, HG $\rightarrow$ PT was higher in the static single tone condition $\left(\chi_{\text {diff, } 1}^{2}=4.79, \mathrm{p}=0.03\right)$, while PT $\rightarrow$ STS was lower in the static harmonic-complex condition $\left(\chi^{2}\right.$ diff,1 $\left.=9.74, \mathrm{p}<0.01\right)$. For subject 3 , alSTG $\rightarrow$ PT was higher in the FM harmonic-complex condition $\left(\chi_{\text {diff,1 }}^{2}=3.72, \mathrm{p}=0.05\right)$. For subject 4 , in the right hemisphere, HG $\rightarrow$ PT was lower in the static harmonic-complex and baseline conditions $\left(\chi_{\text {diff, } 1}^{2}=6.27, \mathrm{p}\right.$ $=0.01$ ), while in the left hemisphere, alSTG $\rightarrow$ PT was marginally lower in the FM harmoniccomplex and baseline conditions $\left(\chi_{\text {diff, } 1}^{2}=2.99, \mathrm{p}=0.08\right)$. Condition-specific effects were found for PT $\rightarrow$ STS in both hemispheres, but for different conditions. On the right, PT $\rightarrow$ STS was higher for the static harmonic-complex and baseline conditions $\left(\chi_{\text {diff, } 1}^{2}=5.33, \mathrm{p}=\right.$ 0.02) and, on the left, for the FM single tone condition alone $\left(\chi_{\text {diff, } 1}^{2}=3.86, \mathrm{p}=0.05\right)$. Subject 5 showed higher path strengths for HG $\rightarrow$ STS $\left(\chi_{\text {diff,1 }}^{2}=9.35, \mathrm{p}<0.01\right)$ in the static harmonic-complex and baseline conditions, and lower path strengths for alSTG $\rightarrow$ PT $\left(\chi^{2}\right.$ diff,1 $=4.65, \mathrm{p}=0.03$ ) in both static tone conditions. Finally, subject 6 showed a marginally higher connection for HG $\rightarrow$ alSTG in the FM harmonic-complex and baseline conditions $\left(\chi^{2}\right.$ diff, $1=$ 2.98, $\mathrm{p}=0.08)$, and for PT $\rightarrow$ STS in the static tone and baseline conditions $\left(\chi^{2}\right.$ diff,1 $=4.15, \mathrm{p}$ 
$=0.04$ ). Thus, while some significant condition-specific paths could be demonstrated, they were neither replicable across the two voxel selection approaches, nor across subjects. For completeness, we also relaxed the constraints for the path connections for ill-fitting models and report the resulting path coefficients in Table 2. If we consider all the models (both goodand ill-fitting) still no consistent pattern of condition-specific connectivities emerges. This heterogeneity cannot be ascribed to differences in gender or in handedness across the subjects.

**Table $2 * *$

\section{Discussion}

The models in which the data fitted well suggest significant connections between the primary area (HG) and non-primary areas, including PT and alSTG. Several significant condition-specific effects occurred within these paths. However, the precise pattern of effects and model goodness-of-fit were not consistent across subjects nor was it consistent between the group- and individual-based analyses. The inconsistencies in the SEM results may reflect individual differences in the way that the auditory stimuli are processed as the absolute pattern of activation in the fMRI analysis and the path coefficients in the SEM analysis both varied across subjects. However, on the basis of the current data, it is not possible to determine the extent to which the differences in activation patterns and path connectivities reflect processing differences or differences in the noise of the MR signal that contributes to the variance in the data. One would expect that the group-based analysis might reflect some of the features shown by the individual-based analyses. However, given that this was not the case in this experiment, we do not wish to place a strong interpretation on the results of the group-based analysis. The inconsistencies in condition-specific connectivities generate difficulty in interpreting the observed patterns of stimulus-evoked connectivities. Below, we 
further discuss three possible classes of explanation for the lack of reproducibility in the model fits and in the pattern of connectivities for well-fitting models.

Anatomical model: Lack of generalisation in the condition-specific connectivities across subjects may arise if the anatomical model is incorrectly specified. Although our model is informed by primate neuroanatomy, the architecture of human and primate brains may not be directly analogous. Given that sulcal morphology is a poor marker for functional boundaries, there is also an opportunity for inaccuracies in estimating the locations of functional areas. The anatomical model defined in the present study reflects our best estimation using current neuroanatomical knowledge, but, given the variability in the goodness-of-fit indices, it is unlikely to reflect the true anatomical network, which is likely to be much more complex. Voxel selection played an important role for the success of modelling, because changes to the voxel selection had material effects on the goodness-of-fit of the model and the strength of path coefficients. The effect of voxel selection within the four areas of the SEM result may indicate the presence of functional sub-divisions within each of the areas modelled. For example, PT may encompass at least three different fields (Rivier and Clarke, 1997). Difficulties in the precise attribution of voxels to small functional areas are likely to be met in other sensory cortices where such specific within-modality processing is studied.

Experimental design: The main focus of the current study was on the evaluation and mapping of differential responses within areas of the auditory cortex to basic acoustical features such as harmonicity and FM. Peak voxels selected were those that displayed the greatest response to all tone stimuli relative to the baseline (using the t-contrast) and so the auditory network was activated by all tone stimuli to a greater or lesser extent. The SEM may therefore evaluate connectivity differences between those voxels having the strongest non-specific auditory functional activation rather than between those having a strong differential stimulus response. Consequently, the ability to demonstrate stimulus-evoked changes in the effective 
connectivity within this network is likely to rely on subtle differences in the correlation matrix.

Necessity for a good model fit: A good-fitting unconstrained model indicates that the proposed anatomical network provides an appropriate explanation for the data and provides a conservative basis for seeking condition-specific connectivities. Evaluation of conditionspecific differences in the path coefficients are based on finding a significant difference between the fit indices for the unconstrained (assuming equality across conditions) and the constrained (assuming a difference across conditions) models. Constraining a model generally improves model fit, but its significance depends on the magnitude of the change in the $\chi^{2}$ diff value. Paradoxically, an ill-fitting unconstrained model, with a high $\chi^{2}$ value, provides much more scope for freeing model parameters and hence for significantly improving the model fit in the constrained version of the model. Consequently, for an ill-fitting unconstrained model, condition-specific effects are more likely, but may not be robust. In contrast, for an unconstrained model that already provides a good fit to the data, there is less scope for further improvement to the model fit by allowing the path coefficients to vary across conditions. Thus, any condition-specific effects that emerge are more likely to be genuine. While some researchers continue to interpret the parameters and to infer functional significance for stimulus-specific connectivities in models that do not give good overall fit, our more cautious approach would be to reject models that do not consistently fit the data well across subjects. Although by rejecting ill-fitting models we do not interpret all statistically significant path coefficients (elevating the risk of Type I error), this conservatism prevents us from drawing conclusions about stimulus-induced connectivity that are more appropriately attributed to subject-specific effects (Type II error). 


\section{Conclusion}

Using SEM, the fMRI data reported here provided a good fit to the anatomical model in seven out of the 14 cases studied. Model interpretations were variable across subjects depending on the analysis used (group or individual) and on the voxel selection procedure. Other differences in the correlation structure may arise from the sparse temporal sampling of the response. Despite these specific obstacles, our finding of 50\% good model fits leads us to be cautiously optimistic about the future contribution of connectivity analyses to hearing research.

\section{Acknowledgements}

MR Scanning was provided by the Wellcome Department of Cognitive Neurology, London. 


\section{References}

Bentler, P. M. 1992: Structural equation models in medical research. Statistical Methods in Medical Research 1(3): 159-181.

Büchel, C., Coull, J. T. and Friston, K. J. 1999: The predictive value of changes in effective connectivity for human learning. Science 283(5407): 1538-1541.

Büchel, C. and Friston, K. J. 1997: Modulation of connectivity in visual pathways by attention: Cortical interactions evaluated with structural equation modelling and fMRI. Cerebral Cortex 7(8): 768-778.

Bullmore, E., Horwitz, B., Honey, G., Brammer, M., Williams, S. and Sharma, T. 2000: How Good Is Good Enough in Path Analysis of fMRI Data? NeuroImage 11: 289-301.

Fitzpatrick, K. A. and Imig, T. J. 1980: Auditory cortico-cortical connections in the Owl Monkey. The Journal of Comparative Neurology 192: 589-610.

Galaburda, A. and Sanides, F. 1980: Cytoarchitectonic organization of the human auditory cortex. Journal of Comparative Neurology 190: 597-610.

Hackett, T. A., Stepniewska, I. and Kaas, J. H. 1996: Subdivisions of auditory cortex and ipsilateral cortical connections of the parabelt auditory cortex in macaque monkeys. The Journal of Comparative Neurology 394(4): 475-495.

Hadjikhani, N., Liu, A. K., Dale, A. M., Cavanagh, P. and Tootell, R. B. H. 1998: Retinotopy and colour sensitivity in human visual cortical area V8. Nature Neuroscience 1: 235-241.

Hall, D. A., Johnsrude, I. S., Goncalves, M. S., Haggard, M. P., Palmer, A. R., Summerfield, A. Q., Akeroyd, M. A. and Frackowiak, R. S. J. 2000a: Hierarchical processing in the auditory cortex? fMRI mapping of responses to spectral and temporal complexity. European Journal of Neuroscience 12: 494-494.

Hall, D. A., Summerfield, A. Q., Gonçalves, M. S., Foster, J. R., Palmer, A. R. and Bowtell, R. W. 2000b: Time-course of the auditory BOLD response to scanner noise. Magnetic Resonance in Medicine 43(4): 601-606.

Haugton, D. M. A., Oud, J. H. L. and Jansen, R. A. R. G. 1997: Information and other criteria in Structural Equation Model selection. Communications in Statistics-Simulation and Computation 26(4): 1477-1516.

Hayduk, L. A. 1987. Structural Equation Modeling with LISREL - Essential and Advances. The Johns Hopkins University Press, Baltimore and London.

Horwitz, B., McIntosh, A. R., Haxby, J. V., Furey, M., Salerno, J. A., Schapiro, M. B., Rapoport, S. I. and Grady, C. L. 1995: Network analysis of PET-mapped visual pathways in Alzheimer type dementia. NeuroReport 6(17): 2287-2292.

Jaccard, J. and Wan, C. K. 1996. LISREL approaches to interaction effects in multiple regression. Sage Publications, Thousand Oaks, CA. 
Jennings, J. M., McIntosh, A. R. and Kapur, S. 1998: Mapping neural interactivity onto regional activity: An analysis of semantic processing and response mode interactions. Neuroimage 7(3): 244-254.

MacCallum, R. C. 1995. Model Specification: Procedures, Strategies, and Related Issues. In Structural Equation Modelling: Concepts, Issues, and Applications. (R. H. Hoyle, Eds.), 1636. Sage Publications, Inc., Thousand Oaks, CA.

McIntosh, A. R. and Gonzalez-Lima, F. 1991: Structural modeling of functional neural pathways mapped with 2-deoxyglucose:effects of acoustic startle habituation on the auditory system. Brain Research 547: 295-302.

McIntosh, A. R. and Gonzalez-Lima, F. 1993: Network analysis of functional auditory pathways mapped with fluorodeoxyglucose: associative effects of a tone conditioned as a Pablovian excitor or inhibitor. Brain Research 627: 129-140.

McIntosh, A. R. and Gonzalez-Lima, F. 1994a: Network Interactions Among Limbic Cortices, Basal Forebrain, and Cerebellum Differentiate a Tone Conditioned as a Pavlovian Excitor or Inhibitor: Fluorodeoxyglucose Mapping and Covariance Structural Modeling. Journal of Neurophysiology 72(4): 1717-1733.

McIntosh, A. R. and Gonzalez-Lima, F. 1994b: Structural Equation Modeling and Its Application to Network Analysis in Functional Brain Imaging. Human Brain Mapping 2: 222.

McIntosh, A. R. and Gonzalez-Lima, F. 1995: Functional network interactions between parallel auditory pathways during Pavlovian conditioned inhibition. Brain Research 683: 228241.

McIntosh, A. R., Grady, C. L., Ungerleider, L. G., Haxby, J. V., Rapoport, S. I. and Horwitz, B. 1994: Network Analysis of Cortical Visual Pathways Mapped with PET. Journal of Neuroscience 14(2): 655-666.

Moore, B. C. J., Glasberg, B. R. and Baer, T. 1997: A model for the prediction of thresholds, loudness and partial loudness. Journal of the Audio Engineering Society 45: 224-240.

Palmer, A. R., Bullock, D. C. and Chambers, J. D. 1998: A high-output, high-quality sound system for use in auditory fMRI. NeuroImage 7: S359.

Raykov, T. and Widaman, K. F. 1995: Issues in Applied Structural Equation Modeling Research. Structural Equation Modeling 2(4): 289-318.

Rivier, F. and Clarke, S. 1997: Cytochrome Oxidase, Acetylcholinesterase, and NADPHDiaphorase Staining in Human Supratemporal and Insular Cortex: Evidence for Multiple Auditory Areas. Neuroimage 6: 228-304. 
Figure 1 - Representation of four key centres of activation in each hemisphere in the anatomical model. HG, the primary auditory region on Heschl's gyrus; PT, the planum temporale; alSTG, anterolateral superior temporal gyrus; STS, the dorsal bank of the superior temporal sulcus. White arrows denote the hypothesised links between these areas. The anatomical model shown here is for the left hemisphere, but applies equally to the right. 
Table 1 - Strength of the standardised path coefficients between anatomical areas for the goodfitting, unconstrained models.

Coefficients are reported for the group-based modelling (right hemisphere only) and for the individual-based modelling (subjects 2-6, left and right hemispheres where appropriate). Coefficients were tested for significance against all other conditions ( $p<0.1)$ with the exact significance of these condition-specific connectivities being reported in the Results. The nature of these differences are denoted by the superscripted labels, described below.

${ }^{1}$ Higher path coefficient for right HG $\rightarrow$ PT in the static harmonic-complex condition for the group model.

${ }^{2}$ Lower path coefficient for right PT $\rightarrow$ STS in both static single tone conditions for the group model.

${ }^{3}$ Subject 2, higher path coefficient for left HG $\rightarrow$ PT in the static single tone condition.

${ }^{4}$ Subject 2, lower path coefficient for left PT $\rightarrow$ STS in the static harmonic-complex condition.

${ }^{5}$ Subject 3, higher path coefficient for right alSTG $\rightarrow$ PT in the FM harmonic-complex condition.

${ }^{6}$ Subject 4, lower path coefficient for right HG $\rightarrow$ PT in the static harmonic-complex and baseline conditions.

${ }^{7}$ Subject 4, lower path coefficient the left alSTG $\rightarrow$ PT in the FM harmonic-complex and baseline conditions.

${ }^{8}$ Subject 4, higher path coefficient for right PT $\rightarrow$ STS in the static harmonic-complex and baseline conditions.

${ }^{9}$ Subject 4, higher path coefficient for left PT $\rightarrow$ STS in the FM single tone condition.

${ }^{10}$ Subject 5, higher path coefficient for left HG $\rightarrow$ STS in the static harmonic-complex and baseline conditions. 
${ }^{11}$ Subject 5, lower path coefficient for left alSTG $\rightarrow$ PT in both static tone conditions.

${ }^{12}$ Subject 6, higher path coefficient for right HG $\rightarrow$ alSTG in the FM harmonic-complex and baseline conditions.

${ }^{13}$ Subject 6, higher path coefficient for right PT $\rightarrow$ STS in the static single tone and baseline conditions. 
Table 2 - Strength of the standardised path coefficients between anatomical areas for the poorfitting, unconstrained models.

Coefficients are reported for the group-based modelling (left hemisphere only) and for the individual-based modelling (subjects 2-6, left and right hemispheres where appropriate). 


\begin{tabular}{|c|c|c|c|c|c|c|c|c|c|c|c|}
\hline & & $\mathrm{HG} \rightarrow$ & alSTG & $\mathrm{HG}-$ & $\rightarrow \mathrm{PT}$ & alSTG & $\rightarrow \mathrm{PT}$ & $\mathrm{HG}$ & STS & $\mathrm{PT} \rightarrow$ & STS \\
\hline Tone & Subject & Right & Left & Right & Left & Right & Left & Right & Left & Right & Left \\
\hline & 2 & & 0.710 & & $0.575^{3}$ & & -0.146 & & 0.181 & & 0.223 \\
\hline & 3 & 0.347 & & 0.290 & & 0.174 & & 0.248 & & 0.164 & \\
\hline Static & 4 & 0.222 & 0.626 & 0.461 & 0.200 & 0.191 & 0.474 & 0.194 & 0.089 & 0.021 & 0.061 \\
\hline Single & 5 & & 0.144 & & 0.353 & & $0.133^{11}$ & & 0.137 & & 0.501 \\
\hline Tone & 6 & 0.015 & & 0.055 & & -0.008 & & 0.086 & & $0.448^{13}$ & \\
\hline & Group & 0.774 & & 0.162 & & 0.484 & & 0.180 & & $-0.017^{2}$ & \\
\hline & 2 & & 0.839 & & 0.247 & & 0.072 & & 0.250 & & $-0.370^{4}$ \\
\hline & 3 & 0.186 & & 0.414 & & -0.069 & & 0.307 & & -0.009 & \\
\hline Harmonic & 4 & 0.437 & 0.495 & $0.221^{6}$ & 0.362 & 0.258 & 0.352 & -0.167 & 0.157 & $0.363^{8}$ & 0.136 \\
\hline Complex & 5 & & 0.055 & & 0.297 & & $0.205^{11}$ & & $0.421^{10}$ & & 0.403 \\
\hline Tone & 6 & 0.008 & & 0.281 & & 0.031 & & 0.285 & & 0.220 & \\
\hline & Group & 0.727 & & $0.265^{1}$ & & 0.286 & & 0.135 & & $0.063^{2}$ & \\
\hline & 2 & & 0.871 & & 0.326 & & 0.229 & & 0.485 & & -0.134 \\
\hline FM & 3 & 0.283 & & 0.359 & & -0.018 & & 0.089 & & 0.238 & \\
\hline Single & 4 & 0.409 & 0.566 & 0.532 & 0.290 & 0.106 & 0.369 & 0.043 & 0.143 & 0.039 & $0.312^{9}$ \\
\hline Tone & 5 & & 0.252 & & 0.298 & & 0.393 & & 0.185 & & 0.478 \\
\hline & 6 & 0.103 & & 0.274 & & 0.027 & & 0.068 & & 0.135 & \\
\hline & Group & 0.761 & & 0.182 & & 0.393 & & 0.202 & & 0.256 & \\
\hline & 2 & & 0.826 & & 0.169 & & 0.049 & & 0.518 & & -0.219 \\
\hline Harmonic & 3 & 0.202 & & 0.148 & & $0.273^{5}$ & & 0.231 & & 0.322 & \\
\hline & 4 & 0.374 & 0.523 & 0.335 & 0.438 & 0.263 & $0.216^{7}$ & -0.090 & 0.137 & 0.106 & 0.102 \\
\hline Tone & 5 & & 0.200 & & 0.522 & & 0.300 & & 0.052 & & 0.474 \\
\hline & 6 & $0.189^{12}$ & & 0.149 & & 0.149 & & 0.274 & & 0.154 & \\
\hline & Group & 0.769 & & 0.007 & & 0.544 & & 0.165 & & 0.157 & \\
\hline & 2 & & 0.764 & & -0.030 & & 0.237 & & 0.344 & & -0.177 \\
\hline Silent & 3 & 0.276 & & 0.531 & & -0.051 & & 0.417 & & 0.102 & \\
\hline & 4 & 0.385 & 0.487 & $0.077^{6}$ & 0.335 & 0.174 & $0.221^{7}$ & -0.093 & 0.170 & $0.334^{8}$ & 0.051 \\
\hline & 5 & & 0.239 & & 0.331 & & 0.249 & & $0.356^{10}$ & & 0.250 \\
\hline & 6 & $0.247^{12}$ & & 0.259 & & 0.211 & & 0.102 & & $0.388^{13}$ & \\
\hline & Group & 0.735 & & 0.054 & & 0.459 & & 0.086 & & 0.170 & \\
\hline
\end{tabular}


TABLE 2

Strength of the Standardized Path Coefficients between Anatomical Areas for the Poor-Fitting Unconstrained Models

\begin{tabular}{|c|c|c|c|c|c|c|c|c|c|c|c|}
\hline \multirow[b]{2}{*}{ Condition } & \multirow[b]{2}{*}{ Subject } & \multicolumn{2}{|c|}{$\mathrm{HG} \rightarrow$ alSTG } & \multicolumn{2}{|c|}{$\mathrm{HG} \rightarrow \mathrm{PT}$} & \multicolumn{2}{|c|}{ alSTG $\rightarrow$ PT } & \multicolumn{2}{|c|}{$\mathrm{HG} \rightarrow \mathrm{STS}$} & \multicolumn{2}{|c|}{$\mathrm{PT} \rightarrow \mathrm{STS}$} \\
\hline & & Right & Left & Right & Left & Right & Left & Right & Left & Right & Left \\
\hline \multirow[t]{6}{*}{ Static single tone } & Group & & 0.45 & & 0.50 & & 0.16 & & 0.04 & & 0.09 \\
\hline & 1 & 0.61 & 0.36 & 0.53 & 0.58 & 0.24 & 0.23 & 0.00 & 0.00 & 0.46 & 0.48 \\
\hline & 2 & 0.54 & & 0.19 & & 0.29 & & 0.32 & & 0.16 & \\
\hline & 3 & & 0.28 & & 0.54 & & 0.03 & & 0.14 & & 0.35 \\
\hline & 5 & 0.18 & & 0.08 & & 0.30 & & -0.06 & & 0.53 & \\
\hline & 6 & & -0.06 & & 0.39 & & -0.02 & & -0.14 & & 0.05 \\
\hline \multirow{6}{*}{$\begin{array}{l}\text { Static harmonic } \\
\text { complex tone }\end{array}$} & Group & & 0.46 & & 0.62 & & 0.09 & & 0.04 & & 0.21 \\
\hline & 1 & 0.56 & 0.18 & 0.66 & 0.42 & 0.10 & 0.41 & 0.17 & -0.06 & -0.03 & 0.44 \\
\hline & 2 & 0.54 & & 0.22 & & 0.29 & & 0.22 & & -0.11 & \\
\hline & 3 & & 0.09 & & 0.58 & & 0.04 & & 0.31 & & 0.24 \\
\hline & 5 & 0.01 & & 0.18 & & 0.19 & & 0.15 & & 0.19 & \\
\hline & 6 & & 0.07 & & 0.52 & & -0.05 & & 0.49 & & -0.25 \\
\hline \multirow[t]{6}{*}{ FM single tone } & Group & & 0.54 & & 0.54 & & 0.27 & & 0.07 & & 0.23 \\
\hline & 1 & 0.72 & 0.28 & 0.53 & 0.48 & 0.23 & 0.26 & 0.40 & 0.06 & 0.03 & 0.56 \\
\hline & 2 & 0.68 & & 0.48 & & 0.08 & & 0.10 & & 0.27 & \\
\hline & 3 & & 0.23 & & 0.51 & & 0.01 & & 0.05 & & 0.29 \\
\hline & 5 & 0.13 & & 0.23 & & 0.22 & & 0.01 & & 0.27 & \\
\hline & 6 & & 0.30 & & 0.42 & & 0.26 & & 0.10 & & 0.02 \\
\hline \multirow{6}{*}{$\begin{array}{l}\text { FM harmonic } \\
\text { complex tone }\end{array}$} & Group & & 0.53 & & 0.53 & & 0.18 & & 0.11 & & 0.16 \\
\hline & 1 & 0.74 & 0.26 & 0.64 & 0.50 & 0.14 & 0.37 & 0.28 & 0.22 & 0.05 & 0.34 \\
\hline & 2 & 0.55 & & 0.16 & & 0.46 & & 0.10 & & 0.29 & \\
\hline & 3 & & 0.19 & & 0.44 & & 0.24 & & 0.14 & & 0.47 \\
\hline & 5 & 0.14 & & 0.14 & & -0.02 & & -0.06 & & 0.35 & \\
\hline & 6 & & -0.03 & & 0.52 & & 0.22 & & 0.00 & & 0.11 \\
\hline \multirow[t]{6}{*}{ Silent baseline } & Group & & 0.53 & & 0.52 & & 0.26 & & 0.07 & & 0.14 \\
\hline & 1 & 0.47 & 0.19 & 0.74 & 0.27 & 0.10 & 0.26 & 0.08 & 0.03 & 0.27 & 0.43 \\
\hline & 2 & 0.68 & & 0.07 & & 0.38 & & 0.18 & & -0.04 & \\
\hline & 3 & & 0.35 & & 0.51 & & 0.27 & & 0.17 & & 0.31 \\
\hline & 5 & 0.23 & & 0.30 & & 0.15 & & -0.03 & & 0.40 & \\
\hline & 6 & & 0.01 & & 0.55 & & 0.20 & & -0.07 & & 0.02 \\
\hline
\end{tabular}




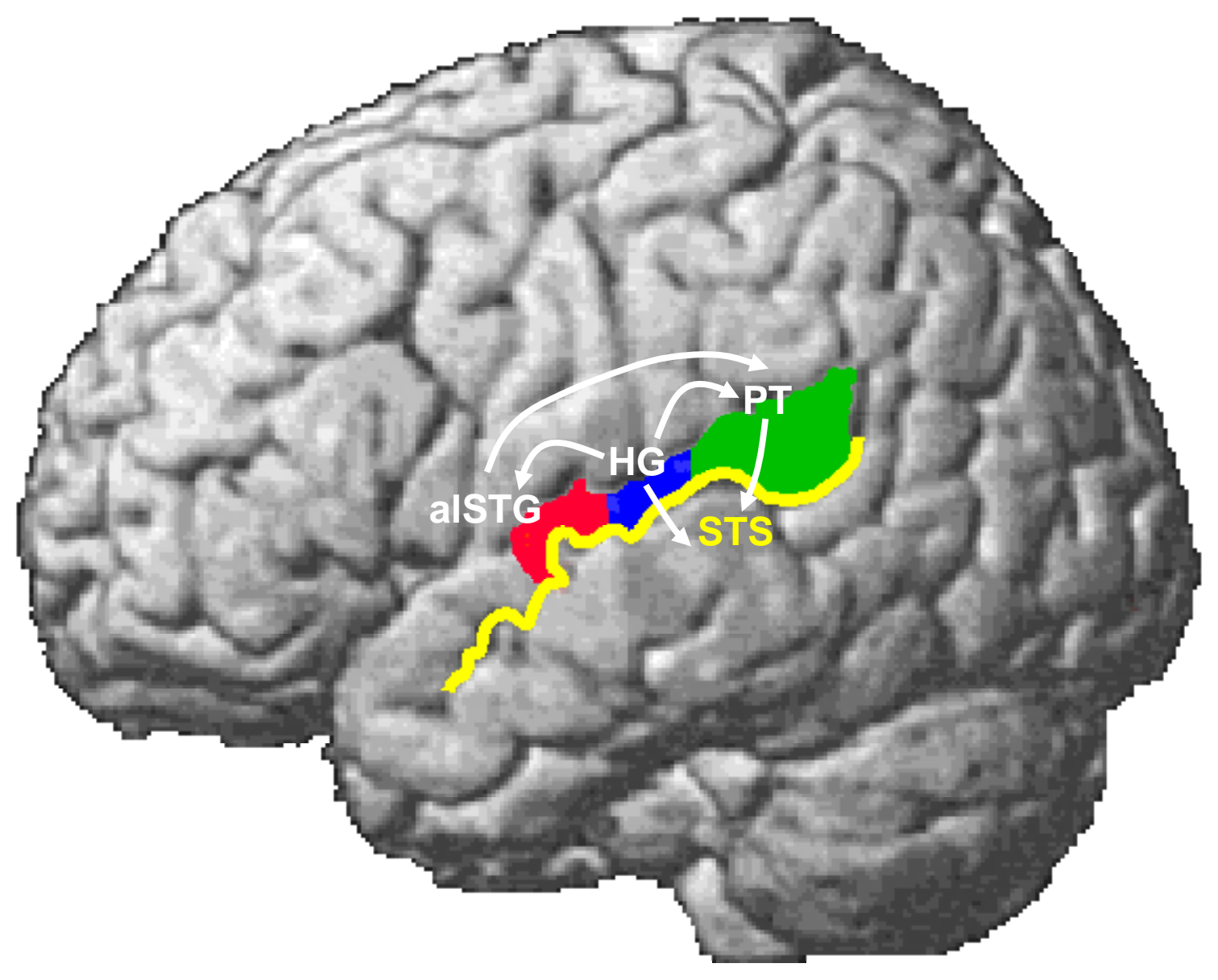

